\title{
The 9 Mile Deposit of the Barama-Mazaruni Greenstone Belt of the Guiana Shield: geochemistry, geochronology and regional significance
}

\author{
Roy Bassoo ${ }^{1,2 *}$ (1), James Brendan Murphy² (1)
}

\begin{abstract}
The granitoid and greenstone-hosted 9 Mile Deposit, located in the Paleoproterozoic Barama-Mazaruni Greenstone Belt of the Guiana Shield, is one of a series of gold deposits within the NW-SE trending Makapa-Kuribrong Shear Zone (MKSZ), which extends from Venezuela, through Guyana, and French Guiana. The 9 Mile Deposit is underlain by the upper section of a shallowly-dipping meta-rhyolite rock, which was intruded by a host granodiorite and subsequently intruded by of a series of mafic dykes. Auriferous quartz veins are associated with the NE-SW thrust which was crosscut by a steep $E$-W shear zone, at least $12 \mathrm{~km}$ in length. Field relationships and lithogeochemical data suggest that the granodiorite was crustally derived and emplaced in a volcanic arc or syn- to late-collisional setting. U-Pb (SHRIMP II) dating of zircons indicates the granodiorite intruded at approximately 2.15, Ga suggesting it is a local representative of a regional suite of syn- to late-tectonic granitoid plutons emplaced during the main phase of Trans-Amazonian Orogeny.
\end{abstract}

KEYWORDS: Guyana; Guiana Shield; Paleoproterozoic; Tectonic evolution; Lithochemistry; Tran-Amazonian Orogeny; Gold deposit.

\section{INTRODUCTION}

Regional syntheses suggest that the Guiana Shield is exposed for over $900,000 \mathrm{~km}^{2}$ along the northern margin of the Amazonian Craton (Fig. 1). There is a broad consensus that the Paleoproterozoic evolution of this craton was dominated by episodes of accretionary tectonics around an Archean core (Tassinari 1997, Cordani \& Teixeira 2007, Daoust et al. 2011, Reis et al. 2000, Fraga et al. 2009, Kroonenberg et al. 2016, Santos et al. 2003). Although exposures are limited, the rocks of the Guiana Shield are thought to be dominated by greenstone belts intruded by a suite of granitoid rocks that became part of the Amazonian Craton during the accretionary orogenic events of the Main Trans-Amazonian (2.26-2.08 Ga) or Late Trans-Amazonian (2.08-1.93 Ga) orogenic events (Vanderhaeghe et al. 1998, Delor et al. 2003). These greenstone belts are host to many orogenic gold deposits and understanding the tectonic processes which formed these rocks will aid in regional gold exploration.

Knowledge of the Guiana Shield and its gold deposits is hindered by inaccessibility and intense tropical weathering, which turns the upper portion of the bedrock into saprolite. Where these occur, detailed studies of fresh exposures provide a rare opportunity to test the validity of the aforementioned regional syntheses. Mineral exploration, fueled by the many occurrences of shear zone hosted gold within and along the Makapa-Kuribrong Shear Zone (Fig. 2), commonly provides new surface exposures and drill core and, therefore, the rare opportunity to examine and analyze relatively fresh samples. This shear zone extends for about $600 \mathrm{~km}$ through Venezuela, Guyana and French Guiana (Voicu et al. 2001). The 9 Mile Deposit, which produced 82,000 ounces of gold from a quartz vein between 1987

1Department of Geosciences, Baylor University - Waco (TX), United States. E-mail: roy_bassoo1@baylor.edu

${ }^{2}$ Department of Earth Sciences, Saint Francis Xavier University - Antigonish, Nova Scotia, Canada. E-mail: bmurphy@stfx.ca

*Corresponding author.

Manuscript ID: 20180072. Received on: 06/20/2018. Approved on: 10/22/2018. 
and 2000 (Veldhuyzen 2010), is one of several epigenetic gold occurrences found within the Makapa-Kuribrong Shear Zone (MKSZ) (Voicu et al. 2001). Although regional studies (e.g., Daoust et al. 2011, Reis et al. 2000) suggest that the geology within the vicinity of the 9 Mile Deposit should be characterized by host and intrusive rocks that are typical of the Guiana Shield, analytical data supporting this contention are sparse (Daoust 2016). In this study, we provided new lithogeochemical analyses of the host and intrusive rocks, as well as a U-Pb (SHRIMP II) zircon age for a granodiorite body. Our data lend support to the validity of regional syntheses, providing insights into orogenic activity in this region, and constraining the age of gold mineralization.

\section{REGIONAL GEOLOGY}

The Guiana Shield (Fig. 1) is divided into four major Paleoproterozoic terranes (Daoust et al. 2011), one Mesoproterozoic terrane and an isolated Archean terrane called the Imataca Complex in Venezuela (Norcross 1997). Crustal development in the Guiana Shield between 2.26 Ga $-2.13 \mathrm{Ga}$ (Tab. 1) created a series of greenstone belts, associated gneisses and amphibolites of the Maroni-Itacaiunas belt (Tassinari et al. 1997). These greenstone belts typically consist of submarine low- $\mathrm{K}$ tholeiitic basalts at the base, overlain by intermediate to felsic volcanic and chemical sedimentary rocks (Gibbs \& Barron 1993, Norcross 1997). Field relationships, combined with recent U-Pb

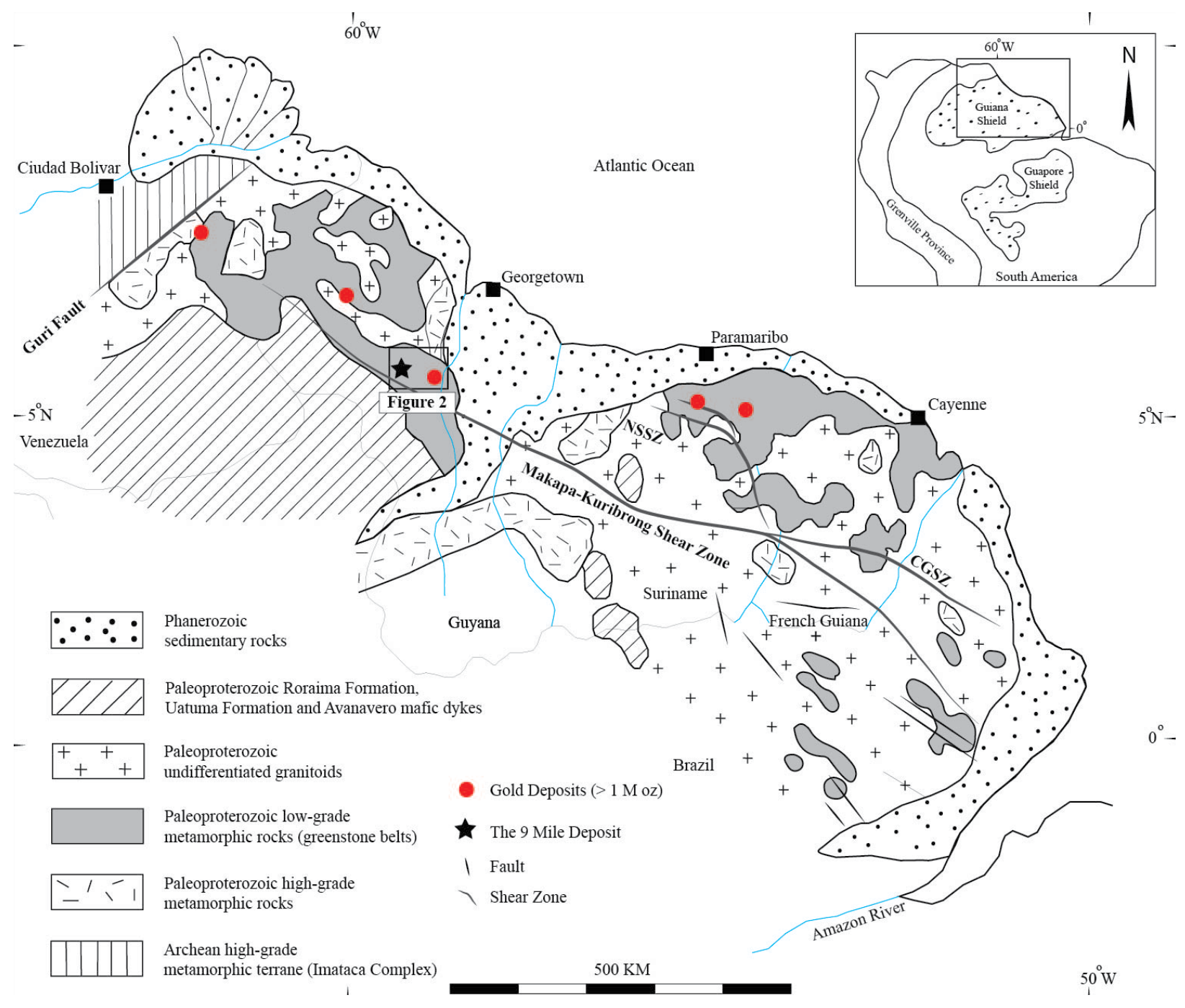

CGSZ: Central Guiana Shear Zone; NSSZ: Northern Suriname Shear Zone.

Figure 1. Simplified geologic map of the Guiana Shield (modified from Voicu 2001, Daoust et al. 2011, Delor et al. 2003, Guiraud et al. 2017). 
radiometric data, suggest the greenstone belts underwent several episodes of deformation, intrusion and metamorphism between ca. $2.26 \mathrm{Ga}-2.08 \mathrm{Ga}$, followed by cooling between $2.08 \mathrm{Ga}-1.93 \mathrm{Ga}$ (Tab. 1). These events are interpreted to reflect the expression of the Trans-Amazonian Orogeny in the eastern Guiana Shield (Gibbs \& Barron 1993, Cordani \& Brito Neves 1982, Norcross 1997, Daoust et al. 2011). Two major episodes of granitoid intrusive activity have been documented in the Guiana Shield. The first episode produced syntectonic granitoids that intruded into the greenstone belt rocks (Norcross 1997) and was coeval with both cataclastic WNW-ESE and subsequent NW-SE sinistral shear zone deformation (Gibbs \& Barron 1993). The NW-SE shearing produced the MKSZ, as well as the Issano-Appaparu

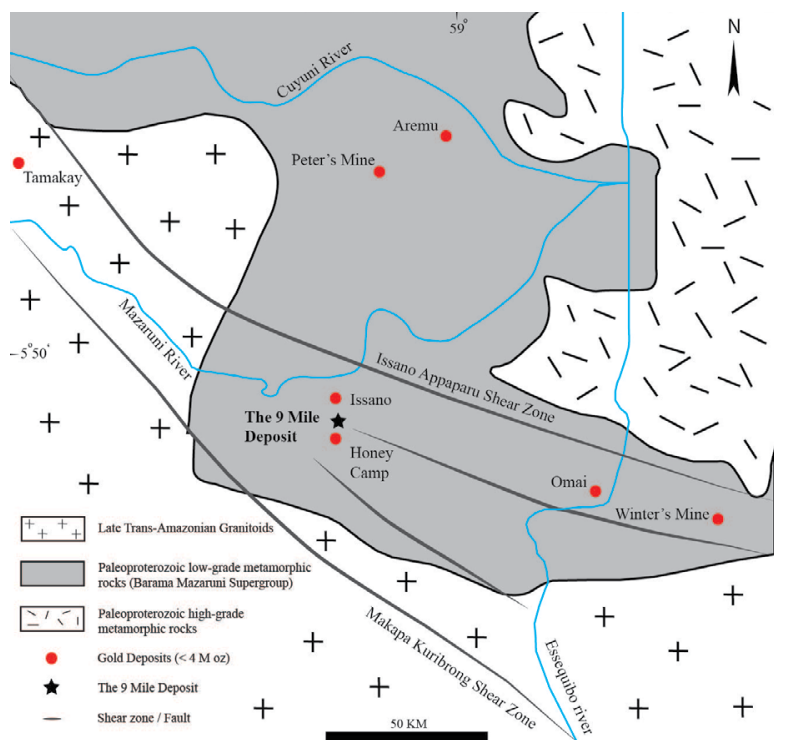

Figure 2. Regional geologic map (modified from Voicu et al. 1999) showing the spatial relationship between gold deposits, the Makapa-Kuribrong Shear Zone (MKSZ) and the Issano Appaparu Shear Zone (IASZ).
Shear Zone (IASZ) (Walrond 1987, Elliott 1992; Fig. 2). Coeval quartz veins and epigenetic gold mineralization typically occur within pelitic and meta-volcanic rocks along the deformed margins of the intrusive rocks (Voicu et al. 2001). The second episode of intrusive activity produced $1.88 \mathrm{Ga}$ - 1.75 Ga post-tectonic K-rich “younger granites” (Daoust et al. 2011). After this episode, the region underwent uplift and erosion, and older rocks are unconformably overlain by the Central Iwokrama Formation of the Uatumã supergroup, which predominantly consists of volcanogenic sedimentary rocks. This formation was intruded by mafic dykes of the Avanavero Suite, dated at $1.79 \pm 0.2 \mathrm{Ga}$, with range of $1.59 \mathrm{Ga}$ to $1.97 \mathrm{Ga}$ (Reis et al. 2000). These rocks were then unconformably overlain by the Roraima Formation, a sequence dominated by interbedded sandstones and conglomerates (Priem et al. 1973, Santos et al. 2003).

\section{LOCAL GEOLOGY}

The tectonic sequence of events in the vicinity of the 9 Mile Deposit is summarized in Table 2. The deposit is hosted by the Paleoproterozoic Barama-Mazaruni Supergroup (Fig. 2), which has a typical greenstone belt stratigraphy (Carter \& Fernandes 1966, Gibbs \& Barron 1993, Norcross 1997, Voicu 1999, Daoust et al. 2011) consisting of tholeiitic basalts and associated mafic-ultramafic dykes and sills, unconformably overlain by intermediate-felsic volcanic rocks that are interlayered with thick sedimentary and volcanogenic-sedimentary rocks. This supergroup is intruded by the syn- to late-tectonic calc-alkaline Trans-Amazonian "granitoids" which range from felsic to intermediate in composition (Vanderhaeghe et al. 1998). The 9 Mile Deposit area (Fig. 3) covers approximately $8 \mathrm{~km}^{2}$ and is underlain by a shallowly dipping and foliated meta-rhyolite with minor foliated carbonate lenses, which was intruded by a coarse-grained granodiorite, as evidenced

Table 1. Tectonic sequence of events of the Barama-Mazaruni formation of the Guiana Shield*.

\begin{tabular}{l|c|c}
\hline Tectonic Episode & Event & U-Pb Age Constraints (Ga) \\
\hline \multirow{2}{*}{$\begin{array}{l}\text { Post Trans-Amazonian } \\
\text { Orogeny }\end{array}$} & Deposition of the Roraima Formation & $1.68-1.57$ \\
\cline { 2 - 3 } & $\begin{array}{c}\text { Deposition of Central Iwokrama Formation felsic volcanogenic } \\
\text { sedimentary rocks and intrusion of post-tectonic granitoids }\end{array}$ & $1.59-1.97$ \\
\hline $\begin{array}{l}\text { Late Trans-Amazonian } \\
\text { Orogeny }\end{array}$ & $\begin{array}{c}\text { Deposition of Muruwa sandstones, siltstones } \\
\text { and conglomerates. Intrusion of Uatumã granites }\end{array}$ & $2.875-1.75$ \\
\hline $\begin{array}{l}\text { Early Trans-Amazonian } \\
\text { Orogeny }\end{array}$ & $\begin{array}{c}\text { Formation of greenstone belt stratigraphy in the Guiana Shield, including } \\
\text { protoliths of gneisses and amphibolites of the Maroni-Itacaiunas belt }\end{array}$ & $2.08-2.93$ \\
\hline
\end{tabular}

${ }^{*}$ Compiled from Daoust et al. (2011), Voicu (1999), Norcross (1997), Gibbs \& Barron (1993), Priem et al. (1973), McConnell \& Williams (1970) and Snelling \& McConnell (1969). Age constraints provide maximum and minimum ages that bracket the formation of rocks. 
by chilled margins in the meta-rhyolite along its contact and meta-rhyolite xenoliths in the granodiorite. An exposed contact between the meta-rhyolite and the granodiorite in the region of the deposit is a NNE-striking thrust fault (Fig. 3), and in core samples intrusive contacts were observed away from the fault. Mafic dykes predominantly strike E-W, and intrusive contacts between the dykes and the granodiorite are observed. Mafic dykes are fine to medium-grained and grey with primary major hornblende and $\mathrm{K}$ feldspar, moderate quartz and plagioclase, minor magnetite and secondary chlorite and plagioclase phenocrysts.

Auriferous quartz veins are concentrated along the thrust fault contact between the meta-rhyolite and the granodiorite, which is evidenced by calcite lineations indicating upthrust of the hanging wall relative to the footwall, and by brecciation of the granodiorite. This thrust fault is oriented at 020/20 (right hand rule, strike/dip) and it is brittle-ductile in type. The thrust fault also contains fractured and stretched euhedral pyrite concentrated in the meta-rhyolite along its contact. A younger, steeply dipping E-W shear zone transects all lithologies and the thrust fault at the deposit site have auriferous quartz veins $(0.05-0.12 \mathrm{~g} / \mathrm{t}$; LaFrance 2010) occurring along its shear plane and along associated en-echelon tension gashes $(0.66-0.76 \mathrm{~g} / \mathrm{t}$; LaFrance 2010$)$ and shear fractures $(316 / 53)$. Within auriferous quartz veins there are fine-grained euhedral pyrite in the center and finegrained subhedral to anhedral stretched and rotated pyrite in the margins. Both forms of pyrite found inside the quartz vein indicate that the quartz veins are coeval with the thrust fault and also part of a sequence of progressive deformation.

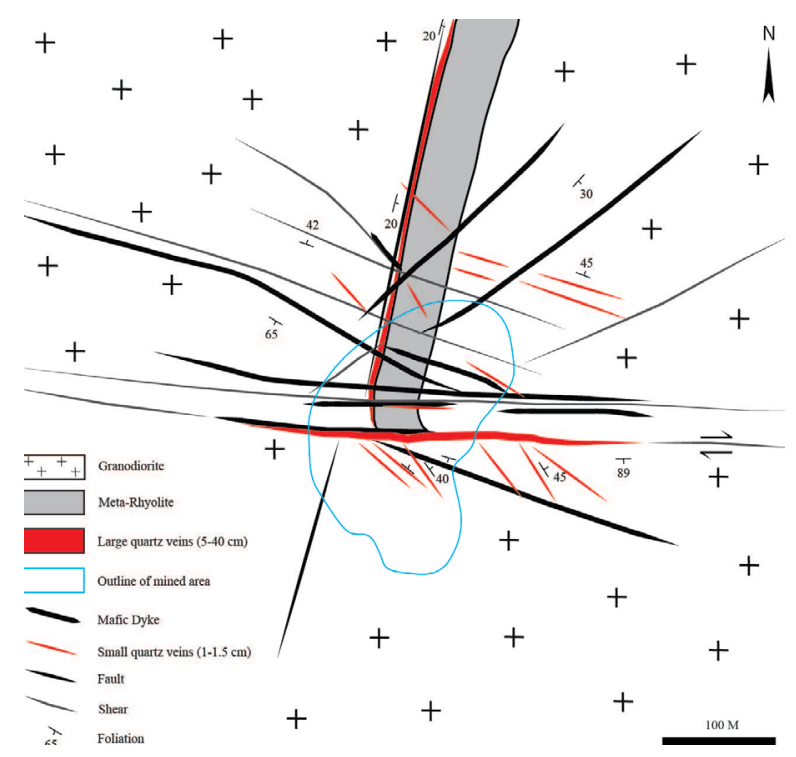

Figure 3. Geological sketch map of the 9 Mile Deposit.

Table 2. Tectonic sequence of events of the 9 Mile Deposit*.

\begin{tabular}{|c|c|c|c|c|}
\hline Tectonic setting & Event & Comments & $\begin{array}{l}\mathrm{U}-\mathrm{Pb} \text { age range }(\mathrm{Ga}) \\
\text { From the literature }\end{array}$ & $\begin{array}{l}\text { U-Pb measured Age (Ga) } \\
\text { This study }\end{array}$ \\
\hline \multirow{3}{*}{$\begin{array}{l}\text { Late } \\
\text { Trans-Amazonian } \\
\text { Orogeny }\end{array}$} & $\begin{array}{l}\text { Intrusion of } \\
\text { mafic dykes }\end{array}$ & $\begin{array}{l}\text { Parallel to E-W and NE-SW } \\
\text { shear foliations }\end{array}$ & $2.07-1.93$ & \\
\hline & E-W shearing & $\begin{array}{l}\text { Auriferous quartz veins } \\
\text { parallel to E-W shear zone } \\
\text { and NW-SE enechelon shear } \\
\text { fractures }\end{array}$ & $2.07-1.93$ & \\
\hline & $\begin{array}{l}\text { Quartz veining } \\
\text { parallel to } \\
\text { NE-SW thrust }\end{array}$ & $\begin{array}{l}\text { Auriferous quartz veins. } \\
\text { Euhedral pyrite and xenoliths } \\
\text { of granodiorite are found } \\
\text { within quartz veins }\end{array}$ & $2.07-1.93$ & \\
\hline \multirow{4}{*}{$\begin{array}{l}\text { Main } \\
\text { Trans-Amazonian } \\
\text { Orogeny }\end{array}$} & $\begin{array}{l}\text { NE-SW thrust } \\
\text { faulting }\end{array}$ & $\begin{array}{l}\text { Thrust faulted contact of } \\
\text { meta-rhyolite and granodiorite }\end{array}$ & $2.11-2.08$ & \\
\hline & $\begin{array}{l}\text { Intrusion of } \\
\text { granodiorite }\end{array}$ & & $2.26-2.13$ & \multirow{3}{*}{$2.142 \pm 0.17-2.147 \pm 0.12$} \\
\hline & $\begin{array}{l}\text { NW-SE shearing } \\
\quad \text { of rhyolite }\end{array}$ & $\begin{array}{l}\text { Marked by stretched and } \\
\text { deformed ankerite and } \\
\text { dolomite }\end{array}$ & $2.26-2.13$ & \\
\hline & $\begin{array}{l}\text { Deposition of } \\
\text { rhyolite }\end{array}$ & & $2.26-2.13$ & \\
\hline
\end{tabular}

${ }^{*}$ Compiled from Gibbs \& Barron (1993), Voicu (1999) and Norcross (1997). Age constraints provide maximum and minimum ages that bracket the formation of rocks. 
Quartz veins have varying dips, ranging from $20^{\circ}$ to vertical. The auriferous quartz veins have three dominant orientations:

- 020/20 (A) (20 - $40 \mathrm{~cm}$ width);

089/89 (B) (15 to $20 \mathrm{~cm}$ width);

$316 / 53$ (5 to $10 \mathrm{~cm}$ width) $\left(\mathrm{B}_{\mathrm{i}}\right)$.

(A) group quartz veins occur along the thrust fault contact between the meta-rhyolite and granodiorite, after the intrusion of the granodiorite. They have grades between 0.39 and $2.73 \mathrm{~g} / \mathrm{t}$ (10 m intervals), including the alteration halo (LaFrance 2010). Individual quartz veins yield an average grade of $9.82 \mathrm{~g} / \mathrm{t}$.

(B) group veins occur within the E-W shear zone and cross cut $(\mathrm{A})$ group veins. $\left(\mathrm{B}_{\mathrm{i}}\right)$ group veins occur along tensional fractures and shear fractures coeval with the E-W shear zone. There are also secondary quartz veins (1 to $1.5 \mathrm{~cm}$ width), which have four dominant orientations: $150 / 61$ and 244/65 (B $\left.\mathrm{B}_{\mathrm{ii}}\right), 222 / 57$ (C) and 13/80 (D). $\left(\mathrm{B}_{\mathrm{ii}}\right)$ group veins are part of a conjugate set and formed along fractures formed by brittle deformation caused by the E-W shear zone. (C) group veins and (D) group veins precipitated along fractures and are less commonly observed. Their field relationship to deformation has not been observed. Secondary quartz veins occur preferentially in granodiorite outcrops. Within the meta-rhyolite and dykes, quartz veining is rare.

The meta-rhyolite is fine-grained and is dominated by primary quartz, plagioclase, $\mathrm{K}$ feldspar, magnetite and hornblende, and secondary chlorite, pyrite and hematite. The granodiorite is coarse-grained and composed of primary plagioclase, quartz, microcline, hornblende, magnetite and biotite with secondary chlorite. Alteration of plagioclase cores to saussurite is common, and in most instances plagioclase and quartz are fractured. Granodiorites have a very locally developed schistosity, characterized by the parallel orientation of muscovite and chlorite along the foliation plane, but no consistent orientation is evident at the macroscale. Adjacent to shear zones and auriferous quartz veins, dynamic recrystallization of quartz, precipitation of muscovite and grain size reduction of minerals are prevalent, and pyrite is locally fragmented, stretched and rotated. Magnetite grains are present on chlorite, likely from excess iron produced by the alteration of biotite.

There are no previously published precise geochronological data available from the study area, until now (Tab. 3). The most relevant precise age data are from rocks adjacent to the Omai gold deposit, which is located approximately $25 \mathrm{~km}$ southeast of the 9 Mile Deposit and within the same gold corridor (Norcross et al. 2000). Ages of 2094

Table 3. $\mathrm{U}$ and $\mathrm{Pb}$ isotopic compositions and ages of zircons from the 9 Mile Deposit granodiorite.

\begin{tabular}{|c|c|c|c|c|c|c|c|c|c|c|c|c|c|}
\hline $\begin{array}{l}\text { Zircon } \\
\text { Spot } \\
\text { Sample }\end{array}$ & $\begin{array}{c}{ }^{206} \mathrm{~Pb} \\
(\%)\end{array}$ & $\begin{array}{c}\mathrm{U} \\
(\mathrm{ppm})\end{array}$ & $\begin{array}{c}\text { Th } \\
\text { (ppm) }\end{array}$ & $\begin{array}{l}{ }^{232} \mathrm{Th} / \\
{ }^{238} \mathrm{U}\end{array}$ & $\begin{array}{c}\mathrm{Pb} \\
(\mathrm{ppm})\end{array}$ & $\begin{array}{c}{ }^{206} \mathrm{~Pb} / 238 \mathrm{U} \\
\text { Age (Ma) } \\
\pm 2 \sigma\end{array}$ & $\begin{array}{c}{ }^{207} \mathrm{~Pb} /{ }^{206} \mathrm{~Pb} \\
\mathrm{Age}(\mathrm{Ma}) \\
\pm 2 \sigma\end{array}$ & $\begin{array}{c}\text { Discordant } \\
\%\end{array}$ & $\begin{array}{c}{ }^{238} \mathrm{U} / \\
{ }^{20} \mathrm{~Pb} \pm 1 \sigma\end{array}$ & $\begin{array}{c}{ }^{207} \mathrm{~Pb} / \\
{ }^{206} \mathrm{~Pb} \pm 1 \sigma\end{array}$ & $\begin{array}{c}{ }^{207} \mathrm{~Pb} / \\
{ }^{235} \mathrm{U} \pm 1 \sigma\end{array}$ & $\begin{array}{c}{ }^{206} \mathrm{~Pb} / \\
{ }^{238} \mathrm{U} \pm 1 \sigma\end{array}$ & $\begin{array}{c}\text { Error } \\
\text { Correction }\end{array}$ \\
\hline $\begin{array}{l}\text { YK } 109 \\
1.1\end{array}$ & 0.19 & 90.00 & 39.40 & 0.45 & 30.60 & $\begin{array}{c}2141 \pm \\
25\end{array}$ & $2147 \pm 27$ & 0.32 & $\begin{array}{c}2.54 \pm \\
1.40\end{array}$ & $\begin{array}{c}0.13 \pm \\
1.50\end{array}$ & $\begin{array}{c}7.26 \pm \\
2.10\end{array}$ & $\begin{array}{c}0.39 \pm \\
1.40\end{array}$ & 0.67 \\
\hline $\begin{array}{l}\text { YK } 109 \\
2.1\end{array}$ & 0.12 & 126.00 & 75.20 & 0.62 & 39.20 & $\begin{array}{c}1988 \pm \\
22\end{array}$ & $2144 \pm 22$ & 7.83 & $\begin{array}{c}2.77 \pm \\
1.30\end{array}$ & $\begin{array}{c}1.33 \pm \\
1.30\end{array}$ & $\begin{array}{c}6.65 \pm \\
1.80\end{array}$ & $\begin{array}{c}0.36 \pm \\
1.30\end{array}$ & 0.71 \\
\hline $\begin{array}{l}\text { YK } 109 \\
3.1\end{array}$ & 0.21 & 115.00 & 52.00 & 0.47 & 39.00 & $\begin{array}{c}2136 \pm \\
26\end{array}$ & $2151 \pm 24$ & 0.71 & $\begin{array}{c}2.55 \pm \\
1.40\end{array}$ & $\begin{array}{l}1.34 \pm \\
1.40\end{array}$ & $\begin{array}{c}7.26 \pm \\
2.00\end{array}$ & $\begin{array}{c}0.39 \pm \\
1.40\end{array}$ & 0.72 \\
\hline $\begin{array}{l}\text { YK } 109 \\
4.1\end{array}$ & 0.12 & 174.00 & 134.00 & 0.79 & 59.10 & $\begin{array}{c}2140 \pm \\
23\end{array}$ & $2151 \pm 18$ & 0.52 & $\begin{array}{c}2.54 \pm \\
1.30\end{array}$ & $\begin{array}{c}0.13 \pm \\
1.10\end{array}$ & $\begin{array}{c}7.27 \pm \\
1.60\end{array}$ & $\begin{array}{c}0.39 \pm \\
1.30\end{array}$ & 0.77 \\
\hline $\begin{array}{l}\text { YK } 109 \\
5.1\end{array}$ & 0.28 & 179.00 & 116.00 & 0.67 & 60.90 & $\begin{array}{c}2147 \pm \\
23\end{array}$ & $2158 \pm 19$ & 0.50 & $\begin{array}{c}2.53 \pm \\
1.30\end{array}$ & $\begin{array}{c}0.13 \pm \\
1.10\end{array}$ & $\begin{array}{c}7.33 \pm \\
1.70\end{array}$ & $\begin{array}{c}0.40 \pm \\
1.30\end{array}$ & 0.76 \\
\hline $\begin{array}{l}\text { YK } 109 \\
6.1\end{array}$ & 0.25 & 82.00 & 36.90 & 0.47 & 27.90 & $\begin{array}{c}2147 \pm \\
28\end{array}$ & $2121 \pm 28$ & -1.21 & $\begin{array}{c}2.53 \pm \\
1.50\end{array}$ & $\begin{array}{c}0.13 \pm \\
1.60\end{array}$ & $\begin{array}{c}7.18 \pm \\
2.20\end{array}$ & $\begin{array}{c}0.40 \pm \\
1.50\end{array}$ & 0.68 \\
\hline $\begin{array}{l}\text { YK } 109 \\
7.1\end{array}$ & 0.10 & 76.00 & 52.00 & 0.71 & 25.40 & $\begin{array}{c}2112 \pm \\
26\end{array}$ & $2099 \pm 28$ & -0.61 & $\begin{array}{c}2.58 \pm \\
1.40\end{array}$ & $\begin{array}{c}0.13 \pm \\
1.60\end{array}$ & $\begin{array}{c}6.95 \pm \\
2.10\end{array}$ & $\begin{array}{c}0.39 \pm \\
1.40\end{array}$ & 0.67 \\
\hline $\begin{array}{l}\text { YK } 109 \\
8.1\end{array}$ & 0.20 & 95.00 & 40.90 & 0.44 & 32.40 & $\begin{array}{c}2143 \pm \\
25\end{array}$ & $2134 \pm 26$ & -0.39 & $\begin{array}{c}2.54 \pm \\
1.40\end{array}$ & $\begin{array}{c}0.13 \pm \\
1.50\end{array}$ & $\begin{array}{c}7.21 \pm \\
2.00\end{array}$ & $\begin{array}{c}0.39 \pm \\
1.40\end{array}$ & 0.68 \\
\hline $\begin{array}{l}\text { YK } 109 \\
9.1\end{array}$ & 0.10 & 179.00 & 96.70 & 0.56 & 60.60 & $\begin{array}{c}2140 \pm \\
23\end{array}$ & $2158 \pm 19$ & 0.86 & $\begin{array}{c}2.54 \pm \\
1.30\end{array}$ & $\begin{array}{c}0.13 \pm \\
1.10\end{array}$ & $\begin{array}{c}7.30 \pm \\
1.70\end{array}$ & $\begin{array}{c}0.39 \pm \\
1.30\end{array}$ & 0.76 \\
\hline $\begin{array}{l}\text { YK } 109 \\
10.1\end{array}$ & 0.54 & 71.00 & 49.60 & 0.73 & 24.00 & $\begin{array}{c}2134 \pm \\
26\end{array}$ & $2156 \pm 31$ & 1.02 & $\begin{array}{c}2.54 \pm \\
1.50\end{array}$ & $\begin{array}{c}0.13 \pm \\
1.80\end{array}$ & $7.27 \pm 2.3$ & $\begin{array}{c}0.39 \pm \\
1.50\end{array}$ & 0.64 \\
\hline
\end{tabular}


$\pm 1 \mathrm{Ma}$ were derived for a dioritic pluton of the Younger Granite intrusive rocks using zircon, titanite and apatite (U-Pb, TIMS) and $2120 \pm 2 \mathrm{Ma}$ for the host meta-rhyolite at the deposit. The $2002 \pm 5 \mathrm{Ma}$ age of gold mineralization at the Omai deposit was derived from $\mathrm{Pb}-\mathrm{Pb}$ dating of hydrothermal rutile and titanite (Norcross et al. 2000). Additionally, the Salamangone gold deposit, which is approximately $900 \mathrm{~km}$ SE of the 9 Mile Deposit in Amapa, Brazil, has a similar marginally sheared intrusive mineralization style (Nogueira et al. 2000). The host tonalite U-Pb crystallization age is dated at $2160 \pm 130 \mathrm{Ma}$, and its estimated mineralization has been dated to $1830 \pm 270 \mathrm{Ma}$ (Nogueira et al. 2000).

\section{GEOCHEMISTRY OF THE 9 MILE DEPOSIT LITHOLOGIES}

Twelve fresh samples (four mafic dykes, seven granodiorites, and one meta-rhyolite) from the 9 Mile Deposit were selected for lithogeochemical analysis using laser ablation inductively coupled plasma mass spectrometry (LA-ICPMS) (Tab. 4). The petrography of each sample analyzed is given in supplementary data. Incipient alteration evident in thin section excluded the use of traditional geochemical plots involving alkali or alkali earth metals. Plots involving $\mathrm{SiO}_{2}$ (Fig. 4) illustrate the compositional differences of the felsic meta-rhyolite and granodiorite with

Table 4. Laser ablation inductively coupled plasma mass spectrometry (LA-ICPMS) trace element analysis data.

\begin{tabular}{|c|c|c|c|c|c|c|c|c|c|c|c|c|c|}
\hline Sample & Rock Type & $\begin{array}{c}\mathrm{SiO}_{2} \\
(\%)\end{array}$ & $\begin{array}{c}\mathrm{Al}_{2} \mathrm{O}_{3} \\
(\%)\end{array}$ & $\begin{array}{c}\mathrm{Fe}_{2} \mathrm{O}_{3} \\
(\%)\end{array}$ & $\begin{array}{c}\text { Mno } \\
(\%)\end{array}$ & $\begin{array}{c}\mathrm{MgO} \\
(\%)\end{array}$ & $\begin{array}{l}\mathrm{CaO} \\
(\%)\end{array}$ & $\begin{array}{c}\mathrm{Na}_{2} \mathrm{O} \\
(\%)\end{array}$ & $\begin{array}{l}\mathrm{K}_{2} \mathrm{O} \\
(\%)\end{array}$ & $\begin{array}{l}\mathrm{TiO}_{2} \\
(\%)\end{array}$ & $\begin{array}{l}\mathrm{P}_{2} \mathrm{O}_{5} \\
(\%)\end{array}$ & $\begin{array}{l}\text { LOI } \\
(\%)\end{array}$ & $\begin{array}{c}\text { Total } \\
(\%)\end{array}$ \\
\hline Sample 1 & Granodiorite & 72.36 & 13.12 & 3.29 & 0.05 & 0.87 & 2.20 & 3.91 & 2.17 & 0.42 & 0.07 & 1.27 & 99.72 \\
\hline Sample 2 & Granodiorite & 73.00 & 12.48 & 4.08 & 0.05 & 0.88 & 1.91 & 3.63 & 1.49 & 0.49 & 0.05 & 1.75 & 99.81 \\
\hline Sample 6 & Mafic Dyke & 52.56 & 15.07 & 10.62 & 0.14 & 6.56 & 6.49 & 2.78 & 1.70 & 0.77 & 0.25 & 3.31 & 100.20 \\
\hline Sample 7 & Mafic Dyke & 52.83 & 15.85 & 9.66 & 0.14 & 4.61 & 6.74 & 2.39 & 3.05 & 0.83 & 0.24 & 2.84 & 99.18 \\
\hline Sample 8 & Mafic Dyke & 52.15 & 14.27 & 10.76 & 0.15 & 7.08 & 7.20 & 1.75 & 2.17 & 0.80 & 0.16 & 3.83 & 100.30 \\
\hline Sample 9 & Granodiorite & 78.10 & 12.29 & 1.30 & 0.02 & 0.13 & 0.62 & 3.55 & 4.00 & 0.13 & 0.01 & 0.80 & 101.00 \\
\hline Sample 10 & Meta Rhyolite & 72.52 & 13.88 & 2.43 & 0.05 & 0.37 & 1.81 & 3.96 & 2.17 & 0.22 & 0.06 & 2.32 & 99.78 \\
\hline Sample 11 & Granodiorite & 71.11 & 13.64 & 3.26 & 0.05 & 1.10 & 2.25 & 2.16 & 2.95 & 0.42 & 0.10 & 3.75 & 100.80 \\
\hline Sample 12 & Granodiorite & 69.95 & 14.24 & 3.26 & 0.05 & 0.95 & 2.29 & 2.61 & 2.85 & 0.43 & 0.09 & 3.58 & 100.30 \\
\hline Sample 13 & Granodiorite & 70.92 & 13.95 & 3.36 & 0.05 & 0.98 & 2.76 & 4.14 & 2.25 & 0.44 & 0.09 & 1.19 & 100.10 \\
\hline Sample 14 & Granodiorite & 69.31 & 13.73 & 3.43 & 0.06 & 1.05 & 3.20 & 1.12 & 3.62 & 0.41 & 0.09 & 4.59 & 100.60 \\
\hline \multirow[t]{2}{*}{ Sample 15} & Mafic Dyke & 54.13 & 14.90 & 8.57 & 0.13 & 4.18 & 7.14 & 3.50 & 2.58 & 0.91 & 0.47 & 2.93 & 99.45 \\
\hline & & $\begin{array}{c}\text { Sc } \\
(\mathrm{ppm})\end{array}$ & $\begin{array}{c}\mathrm{Be} \\
(\mathrm{ppm})\end{array}$ & $\begin{array}{c}\mathrm{V} \\
(\mathrm{ppm})\end{array}$ & $\begin{array}{c}\mathrm{Cr} \\
(\mathrm{ppm})\end{array}$ & $\begin{array}{c}\text { Co } \\
\text { (ppm) }\end{array}$ & $\begin{array}{c}\mathrm{Ni} \\
(\mathrm{ppm})\end{array}$ & $\begin{array}{c}\mathrm{Cu} \\
(\mathrm{ppm})\end{array}$ & $\begin{array}{c}\mathrm{Zn} \\
(\mathbf{p p m})\end{array}$ & $\begin{array}{c}\mathrm{Ga} \\
(\mathrm{ppm})\end{array}$ & $\begin{array}{c}\mathrm{Ge} \\
\text { (ppm) }\end{array}$ & $\begin{array}{c}\text { As } \\
\text { (ppm) }\end{array}$ & $\begin{array}{c}\mathbf{R b} \\
(\mathbf{p p m})\end{array}$ \\
\hline Sample 1 & Granodiorite & 6 & 1 & 37 & $<20$ & 6 & $<20$ & $<10$ & 40 & 17 & 1.3 & $<5$ & 50 \\
\hline Sample 2 & Granodiorite & 6 & 1 & 42 & $<20$ & 7 & $<20$ & $<10$ & 40 & 17 & 1.4 & $<5$ & 36 \\
\hline Sample 6 & Mafic Dyke & 28 & 2 & 196 & 170 & 35 & 60 & 60 & 80 & 16 & 1.8 & $<5$ & 52 \\
\hline Sample 7 & Mafic Dyke & 23 & 2 & 195 & $<20$ & 29 & 30 & 40 & 90 & 18 & 1.7 & $<5$ & 93 \\
\hline Sample 8 & Mafic Dyke & 29 & 1 & 207 & 190 & 38 & 70 & 60 & 80 & 17 & 1.8 & $<5$ & 74 \\
\hline Sample 9 & Granodiorite & 1 & 1 & 10 & $<20$ & 1 & $<20$ & $<10$ & $<30$ & 13 & 1.5 & $<5$ & 69 \\
\hline Sample 10 & Meta Rhyolite & 3 & 2 & 7 & $<20$ & 2 & $<20$ & $<10$ & $<30$ & 17 & 1.6 & $<5$ & 65 \\
\hline Sample 11 & Granodiorite & 6 & 1 & 36 & $<20$ & 6 & $<20$ & $<10$ & 40 & 16 & 1.5 & $<5$ & 68 \\
\hline Sample 12 & Granc & 6 & 1 & 36 & $<20$ & 6 & $<20$ & 0 & 50 & 18 & 1.6 & $<5$ & 68 \\
\hline Sample 13 & Granodiorite & 6 & 1 & 40 & $<20$ & 7 & $<20$ & $<10$ & 40 & 17 & 1.4 & $<5$ & 51 \\
\hline Sample 14 & Granodiorite & 6 & 1 & 35 & $<20$ & 6 & $<20$ & $<10$ & 40 & 16 & 1.7 & $<5$ & 81 \\
\hline Sample 15 & Mafic Dyke & 20 & 2 & 186 & 30 & 26 & 30 & 70 & 90 & 18 & 1.5 & $<5$ & 67 \\
\hline
\end{tabular}


Table 4. Continuation.

\begin{tabular}{|c|c|c|c|c|c|c|c|c|c|c|c|c|c|}
\hline Sample & Rock Type & $\begin{array}{c}\mathrm{SiO}_{2} \\
(\%)\end{array}$ & $\begin{array}{c}\mathrm{Al}_{2} \mathrm{O}_{3} \\
(\%)\end{array}$ & $\begin{array}{c}\mathrm{Fe}_{2} \mathrm{O}_{3} \\
(\%)\end{array}$ & $\begin{array}{c}\text { Mno } \\
(\%)\end{array}$ & $\begin{array}{c}\text { MgO } \\
(\%)\end{array}$ & $\begin{array}{l}\mathrm{CaO} \\
(\%)\end{array}$ & $\begin{array}{c}\mathrm{Na}_{2} \mathrm{O} \\
(\%)\end{array}$ & $\begin{array}{l}\mathrm{K}_{2} \mathrm{O} \\
(\%)\end{array}$ & $\begin{array}{c}\mathrm{TiO}_{2} \\
(\%)\end{array}$ & $\begin{array}{c}P_{2} \mathbf{O}_{5} \\
(\%)\end{array}$ & $\begin{array}{l}\text { LOI } \\
(\%)\end{array}$ & $\begin{array}{c}\text { Total } \\
(\%)\end{array}$ \\
\hline & & $\begin{array}{c}\mathbf{Y} \\
(\mathrm{ppm})\end{array}$ & $\begin{array}{c}\mathrm{Zr} \\
(\mathrm{ppm})\end{array}$ & $\begin{array}{c}\text { Nb } \\
\text { (ppm) }\end{array}$ & $\begin{array}{c}\text { Mo } \\
\text { (ppm) }\end{array}$ & $\begin{array}{c}\mathrm{Ag} \\
(\mathrm{ppm})\end{array}$ & $\begin{array}{c}\text { In } \\
\text { (ppm) }\end{array}$ & $\begin{array}{c}\text { Sn } \\
(\mathbf{p p m})\end{array}$ & $\begin{array}{c}\text { Sb } \\
\text { (ppm) }\end{array}$ & $\begin{array}{c}\text { Cs } \\
\text { (ppm) }\end{array}$ & $\begin{array}{c}\mathrm{Ba} \\
(\mathrm{ppm})\end{array}$ & $\begin{array}{c}\text { La } \\
(\mathrm{ppm})\end{array}$ & $\begin{array}{c}\text { Ce } \\
\text { (ppm) }\end{array}$ \\
\hline Sample 1 & Granodiorite & 18.8 & 178 & 5.9 & $<2$ & 1.3 & $<0.1$ & 1 & $<0.2$ & 0.5 & 531 & 52 & 75.4 \\
\hline Sample 2 & Granodiorite & 8.2 & 223 & 7.3 & $<2$ & 1.5 & $<0.1$ & 2 & $<0.2$ & 0.6 & 461 & 15.5 & 45.5 \\
\hline Sample 6 & Mafic Dyke & 16.4 & 105 & 4.2 & $<2$ & 0.7 & $<0.1$ & $<1$ & $<0.2$ & 0.5 & 980 & 18.7 & 39.5 \\
\hline Sample 7 & Mafic Dyke & 18.1 & 116 & 4.6 & $<2$ & 0.8 & $<0.1$ & $<1$ & $<0.2$ & 0.6 & 1,222 & 22.8 & 43.4 \\
\hline Sample 8 & Mafic Dyke & 15.3 & 119 & 3.9 & $<2$ & 0.8 & $<0.1$ & 1 & $<0.2$ & 0.8 & 924 & 20.3 & 42.8 \\
\hline Sample 9 & Granodiorite & 9.3 & 85 & 7.5 & $<2$ & $<0.5$ & $<0.1$ & 2 & $<0.2$ & 0.4 & 499 & 13.4 & 24.2 \\
\hline Sample 10 & Meta Rhyolite & 16.1 & 170 & 8.4 & $<2$ & 1.1 & $<0.1$ & 1 & $<0.2$ & 1.5 & 511 & 34.3 & 66.6 \\
\hline Sample 11 & Granodiorite & 15.7 & 201 & 7.1 & $<2$ & 1.4 & $<0.1$ & 1 & 0.4 & 1.2 & 511 & 30.7 & 59 \\
\hline Sample 12 & Granodiorite & 16.5 & 203 & 7.3 & $<2$ & 1.4 & $<0.1$ & 1 & 0.3 & 1.4 & 489 & 29.9 & 56.6 \\
\hline Sample 13 & Granodiorite & 15.7 & 183 & 6.4 & $<2$ & 1.2 & $<0.1$ & 1 & $<0.2$ & 0.4 & 550 & 31.1 & 59.4 \\
\hline Sample 14 & Granodiorite & 15.2 & 217 & 7.1 & $<2$ & 1.4 & $<0.1$ & 1 & 0.6 & 1.8 & 535 & 30.6 & 58.7 \\
\hline \multirow[t]{2}{*}{ Sample 15} & Mafic Dyke & 20.1 & 148 & 5.7 & $<2$ & 1.1 & $<0.1$ & 1 & $<0.2$ & 1 & 1,251 & 31.8 & 66.1 \\
\hline & & $\begin{array}{c}\mathrm{Nd} \\
\text { (ppm) }\end{array}$ & $\begin{array}{c}\text { Sm } \\
(\text { ppm) }\end{array}$ & $\begin{array}{c}\text { Eu } \\
(\mathbf{p p m})\end{array}$ & $\begin{array}{c}\text { Gd } \\
(\mathrm{ppm})\end{array}$ & $\begin{array}{c}\text { Tb } \\
\text { (ppm) }\end{array}$ & $\begin{array}{c}\text { Dy } \\
\text { (ppm) }\end{array}$ & $\begin{array}{c}\text { Ho } \\
\text { (ppm) }\end{array}$ & $\begin{array}{c}\mathbf{E r} \\
(\mathbf{p p m})\end{array}$ & $\underset{(\mathrm{ppm})}{\mathrm{Tm}}$ & $\begin{array}{c}\mathrm{Yb} \\
(\mathrm{ppm})\end{array}$ & $\begin{array}{c}\mathbf{L u} \\
(\mathbf{p p m})\end{array}$ & $\begin{array}{c}\text { Hf } \\
\text { (ppm) }\end{array}$ \\
\hline Sample 1 & Granodiorite & 35.5 & 5.29 & 1.1 & 4.3 & 0.61 & 3.29 & 0.66 & 1.86 & 0.273 & 1.82 & 0.286 & 4.1 \\
\hline Sample 2 & Granodiorite & 12.6 & 2.25 & 0.638 & 1.52 & 0.27 & 1.7 & 0.34 & 1.02 & 0.173 & 1.34 & 0.217 & 5 \\
\hline Sample 6 & Mafic Dyke & 22.5 & 5.01 & 1.39 & 4.24 & 0.63 & 3.36 & 0.64 & 1.72 & 0.243 & 1.78 & 0.246 & 2.5 \\
\hline Sample 7 & Mafic Dyke & 24.4 & 5.02 & 1.52 & 4.37 & 0.64 & 3.58 & 0.68 & 1.89 & 0.251 & 1.69 & 0.257 & 2.7 \\
\hline Sample 8 & Mafic Dyke & 23 & 5.1 & 1.35 & 3.96 & 0.59 & 3.27 & 0.61 & 1.66 & 0.232 & 1.52 & 0.242 & 3 \\
\hline Sample 9 & Granodiorite & 10.1 & 1.9 & 0.248 & 1.47 & 0.23 & 1.48 & 0.32 & 1.02 & 0.186 & 1.44 & 0.254 & 3.1 \\
\hline Sample 10 & Meta Rhyolite & 27.7 & 4.78 & 1.3 & 3.59 & 0.56 & 3.15 & 0.6 & 1.66 & 0.241 & 1.62 & 0.239 & 4.1 \\
\hline Sample 11 & Granodiorite & 23.2 & 4.09 & 0.823 & 3.14 & 0.48 & 2.87 & 0.59 & 1.69 & 0.266 & 1.92 & 0.288 & 4.9 \\
\hline Sample 12 & Granodiorite & 23.1 & 3.78 & 0.918 & 3.34 & 0.51 & 2.82 & 0.58 & 1.7 & 0.263 & 1.77 & 0.261 & 4.4 \\
\hline Sample 13 & Granodiorite & 24 & 4.08 & 0.966 & 3.24 & 0.51 & 2.84 & 0.56 & 1.69 & 0.258 & 1.74 & 0.27 & 4.4 \\
\hline Sample 14 & Granodiorite & 22.5 & 4.1 & 0.893 & 2.97 & 0.48 & 2.83 & 0.58 & 1.73 & 0.26 & 1.76 & 0.278 & 4.9 \\
\hline \multirow[t]{2}{*}{ Sample 15} & Mafic Dyke & 34.9 & 7.47 & 1.88 & 5.61 & 0.8 & 4.15 & 0.76 & 2.02 & 0.287 & 1.89 & 0.293 & 3.5 \\
\hline & & $\begin{array}{c}\text { W } \\
\text { (ppm) }\end{array}$ & $\begin{array}{c}\mathrm{Tl} \\
\text { (ppm) }\end{array}$ & $\begin{array}{c}\mathrm{Pb} \\
(\mathrm{ppm})\end{array}$ & $\begin{array}{c}\mathbf{B i} \\
(\mathbf{p p m})\end{array}$ & $\begin{array}{c}\text { Th } \\
\text { (ppm) }\end{array}$ & $\begin{array}{c}\mathrm{U} \\
(\mathrm{ppm})\end{array}$ & $\begin{array}{c}\mathrm{Sr} \\
(\mathbf{p p m})\end{array}$ & $\begin{array}{c}\mathbf{P r} \\
\text { (ppm) }\end{array}$ & $\begin{array}{c}\mathrm{Ta} \\
\text { (ppm) }\end{array}$ & & & \\
\hline Sample 1 & Granodiorite & $<0.5$ & 0.19 & 5 & $<0.1$ & 5.67 & 1.06 & 227 & 10.5 & 0.63 & & & \\
\hline Sample 2 & Granodiorite & 0.7 & 0.16 & 7 & $<0.1$ & 6.12 & 1.07 & 205 & 3.59 & 1.33 & & & \\
\hline Sample 6 & Mafic Dyke & $<0.5$ & 0.21 & 10 & $<0.1$ & 4.38 & 1.4 & 460 & 5.18 & 0.29 & & & \\
\hline Sample 7 & Mafic Dyke & $<0.5$ & 0.46 & 10 & $<0.1$ & 3.66 & 1.31 & 498 & 5.87 & 0.36 & & & \\
\hline Sample 8 & Mafic Dyke & $<0.5$ & 0.3 & 12 & 0.1 & 4.38 & 1.38 & 425 & 5.55 & 0.28 & & & \\
\hline Sample 9 & Granodiorite & $<0.5$ & 0.36 & 12 & $<0.1$ & 11.9 & 3.73 & 65 & 3.03 & 1.7 & & & \\
\hline Sample 10 & Meta Rhyolite & $<0.5$ & 0.27 & 7 & $<0.1$ & 5.23 & 1.37 & 195 & 7.56 & 0.89 & & & \\
\hline Sample 11 & Granodiorite & 34.9 & 0.28 & 6 & $<0.1$ & 6.23 & 1.63 & 106 & 6.59 & 0.92 & & & \\
\hline Sample 12 & Granodiorite & 15.1 & 0.21 & 6 & $<0.1$ & 5.7 & 1.61 & 146 & 6.48 & 0.66 & & & \\
\hline Sample 13 & Granodiorite & $<0.5$ & 0.24 & 6 & $<0.1$ & 5.51 & 1.44 & 256 & 6.78 & 0.73 & & & \\
\hline Sample 14 & Granodiorite & 14 & 0.3 & $<5$ & $<0.1$ & 5.44 & 1.42 & 105 & 6.54 & 0.78 & & & \\
\hline Sample 15 & Mafic Dyke & $<0.5$ & 0.35 & 13 & $<0.1$ & 5.43 & 1.78 & 705 & 8.5 & 0.43 & & & \\
\hline
\end{tabular}


the calc-alkaline mafic dykes (Fig. 5). One meta-rhyolite sample is classified as a dacite, a volcanic equivalent of a granodiorite (Fig. 6), which suggests a similar melt source to the local granodiorites which host the 9 Mile Deposit. The meta-rhyolite also plots in the arc to syn-collisional fields on Nb-Y (Fig. 7) and Ta-Yb (Fig. 8) discrimination plots. The granodiorite samples plot in the magnesian (orogenic) (Fig. 4) and dacite fields (Fig. 6), and on the Nb-Y (Fig. 7) and Ta-Yb (Fig. 8) diagrams, plot in either the volcanic arc or syn-collisional field. Samples 2 and 9 (which are more ferroan) have more elevated $\mathrm{Ta}$ and plot in the syn-collisional field (Fig. 8). The mafic dykes have $\mathrm{SiO}_{2}$ ranging from 54.05 to $56.08 \mathrm{wt} \%$ (on a volatile free basis, Fig. 5). The meta-rhyolite is more enriched in the light rare-earth elements (LREEs) than the granodiorite,

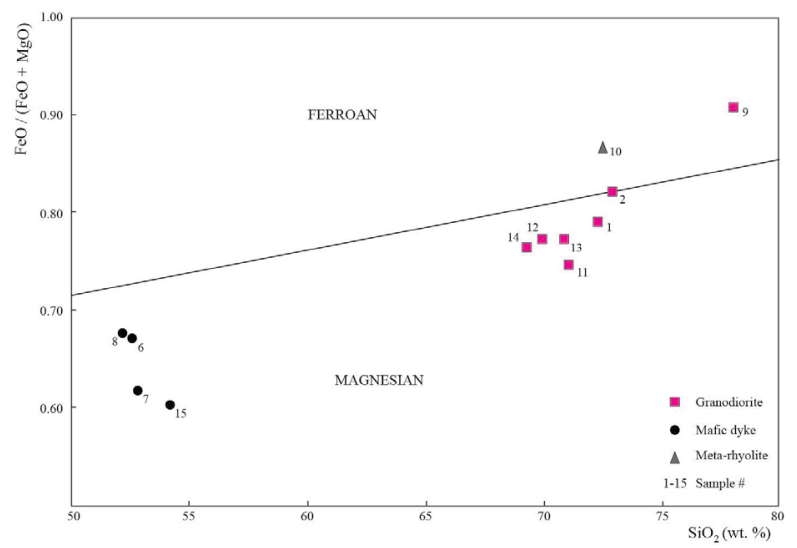

Figure 4. $\mathrm{SiO} 2$ vs. $\mathrm{FeO} /(\mathrm{FeO}+\mathrm{MgO})$ plot. Dividing line between Ferroan and Magnesian after Frost et al. (2001).

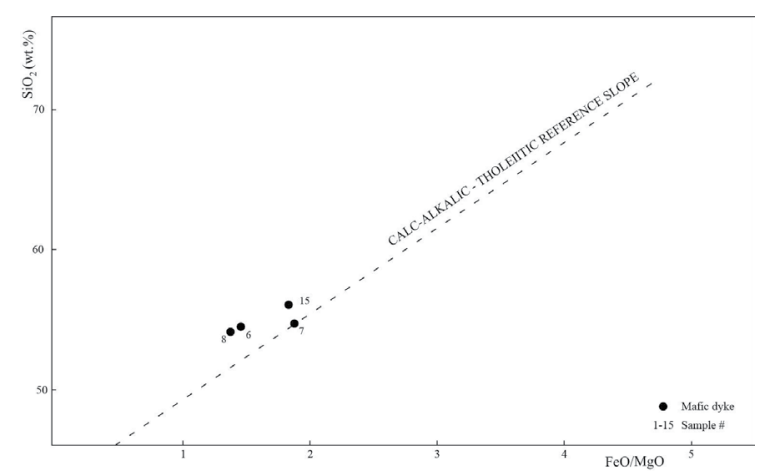

FeOt: total iron oxide.

Figure $5 . \mathrm{FeO}^{\mathrm{t}} / \mathrm{MgO}$ vs. $\mathrm{SiO} 2$ plots for subalkalic rocks (after Miyashiro 1974), showing the slope of mafic dykes of the 9 Mile Deposit. Note that the dashed line is a reference slope. Rocks that have steeper trends than the reference slope are classified as tholeiitic, whereas rocks that have shallower trends than the reference slope are classified as calc-alkalic. Numbers correspond to samples listed in Supplementary Table A1. but also shows depletion in $\mathrm{Ta}, \mathrm{Nb}, \mathrm{P}$ and $\mathrm{Ti}$, which are characteristics typical of crustally-derived magmas (e.g., Pearce et al. 1984). The meta-rhyolite displays moderate LREE enrichment, relatively flat heavy rare earth element (HREE) enrichment, and on a mid ocean ridge basalt (MORB) normalized multi-element plot, has a relatively flat profile, with negative Ta, Nb, P and Ti anomalies (Fig. 9). The granodiorites display moderate LREE enrichment, a variably developed negative Eu (europium) anomaly, and a relatively flat HREE profile (Fig. 10). The mafic dykes plot in the basalt to andesite field (Fig. 6), consistent with thin section petrography. The mafic dykes also plot in the

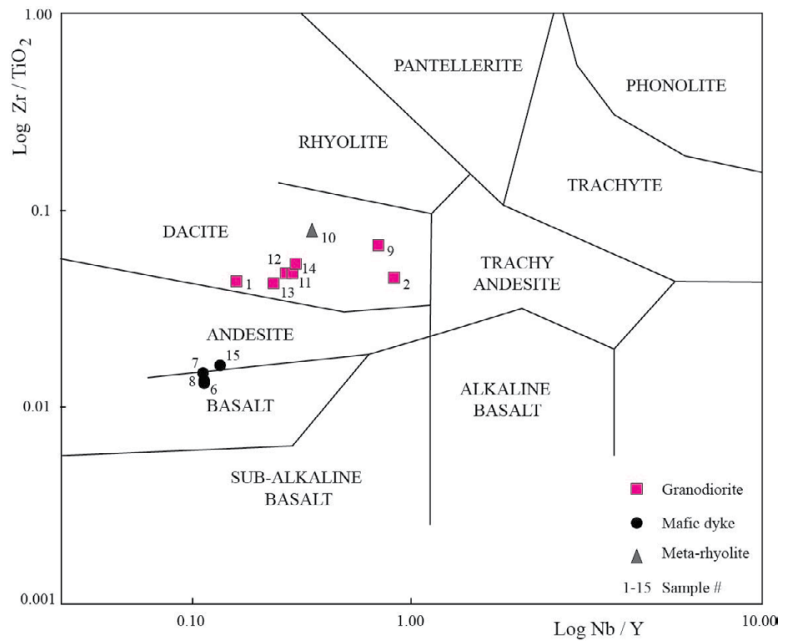

Figure 6. $\mathrm{Zr} / \mathrm{TiO} 2$ vs. $\mathrm{Nb} / \mathrm{Y}$ discrimination diagram (after Winchester \& Floyd 1977). Numbers correspond to samples listed in Supplementary Table A1.

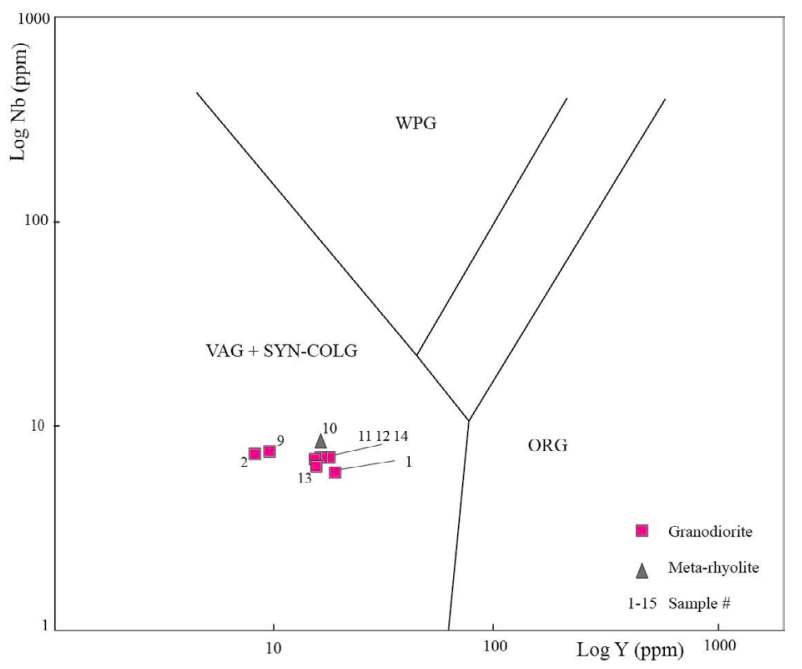

Syn-COLG+VAG: syn-collisional and volcanic arc; ORG: orogenic; WPG: within plate.

Figure 7. Nb vs. Y discrimination diagram (after Pearce et al. 1984). Numbers correspond to samples listed in Supplementary Table A1. 


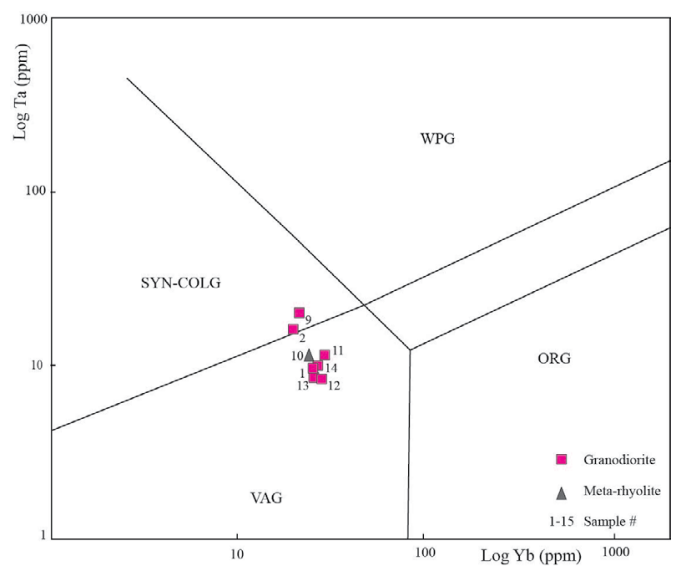

Syn-COLG+VAG: syn-collisional and volcanic arc; ORG: orogenic; WPG: within plate.

Figure 8. Ta vs. Yb discrimination diagram (after Pearce et al. 1984). Numbers correspond to samples listed in Supplementary Table A1.
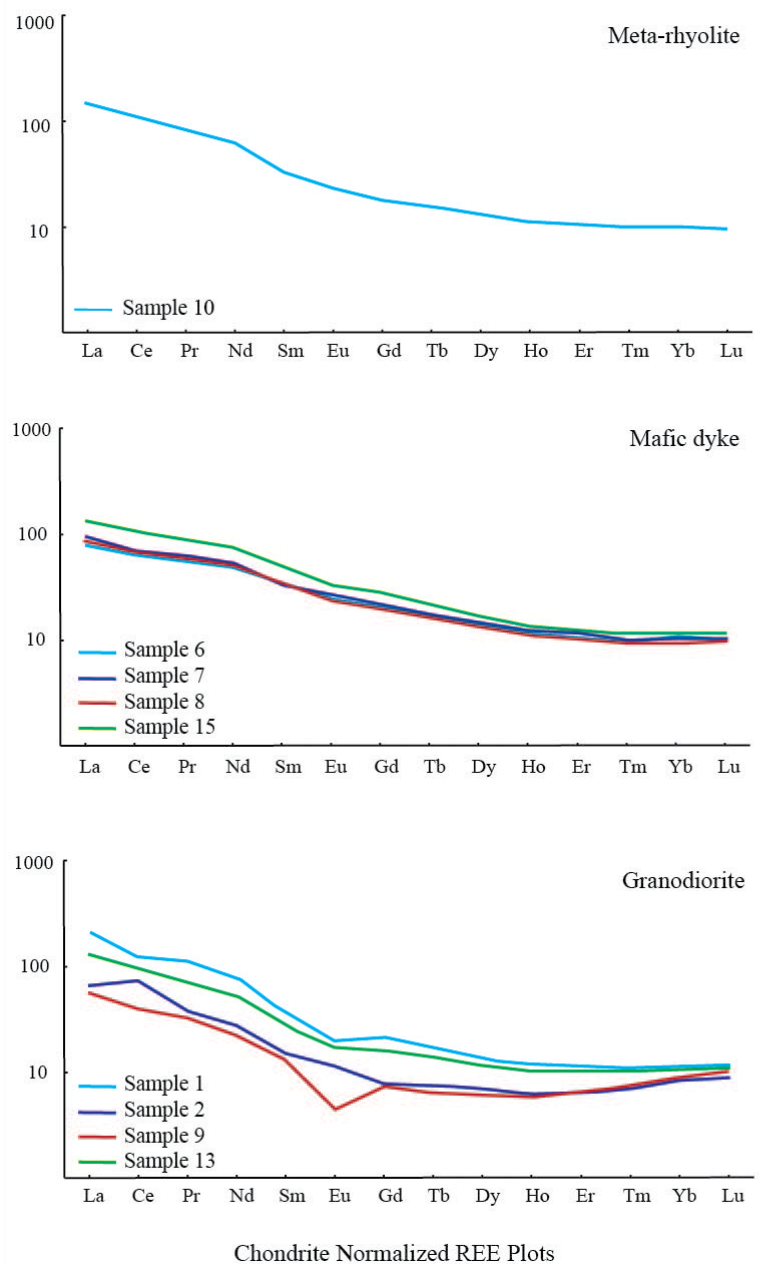

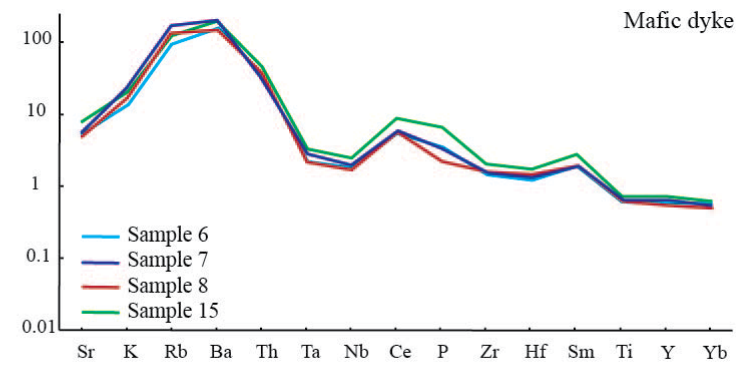

volcanic arc field (Fig. 11) and moderate LREE enrichment (Fig. 9), Zr-Y abundances (Fig. 10) and the shallow $\mathrm{FeO} / \mathrm{MgO}-\mathrm{SiO}_{2}$ slope (Fig. 5) indicates calc-alkaline to transitional mafic dyke compositions and a volcanic arc tectonic setting. Taken together, this suggests that the mafic dykes are part of the regional Orosirian-aged calc-alkaline magmatism known as the Orocaima event in the northern Amazonian Craton (Reis et al. 2000).

\section{U-PB GEOCHRONOLOGY OF THE 9 MILE DEPOSIT GRANODIORITE}

One sample (sample 13) of the host granodiorite at the 9 Mile Deposit was selected for U-Pb dating of zircon grains. Zircon grains were separated and mounted in epoxy together with reference zircons of the TEMORA (Middledale Gabbroic
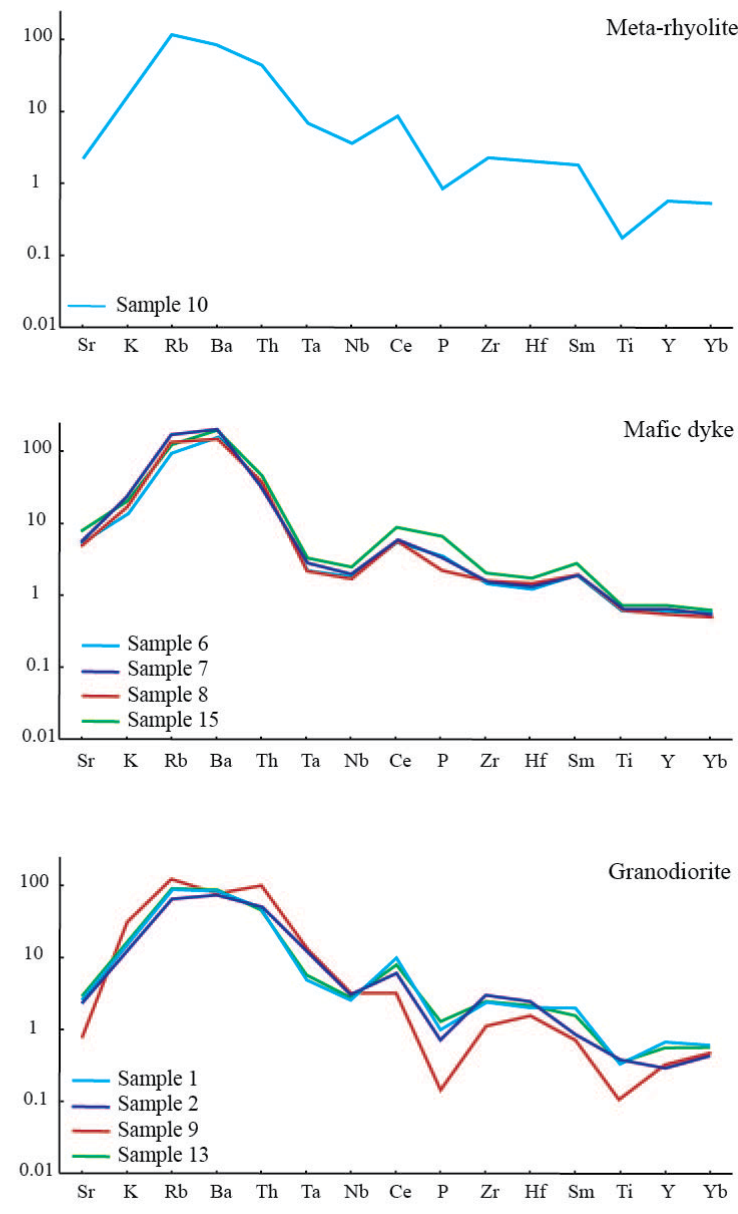

MORB Normalized Multi-Element Plots

Figure 9. Plot of chondrite-normalized (after McDonough \& Sun 1995) rare earth elements (REE) values and mid ocean ridge basalt (MORB)-normalized (after Pearce \& Parkinson 1993) multi-element values of samples of granodiorite, mafic dyke and meta rhyolite. Numbers correspond to samples listed in Supplementary Table A1. 
Diorite, New South Wales, Australia, Black et al. 2003) and 91500 (Geostandard zircon, Wiedenbeck et al. 1995) reference zircons. The grains were sectioned and polished, and cathode luminescence images prepared (Fig. 12). The $\mathrm{U}-\mathrm{Pb}$ analyses of the zircons were conducted by ACME laboratories using SHRIMP-II (Kapusta 2014). The data were reduced in a manner described by Williams (1998) using SQUID software (Ludwig 2000). Uncertainties given for individual analyses (ratios and ages) are at the one sigma level, however the uncertainties in calculated concordia ages are reported as two sigma levels. The AhrensWetherill concordia plots (Wetherill 1956), probability

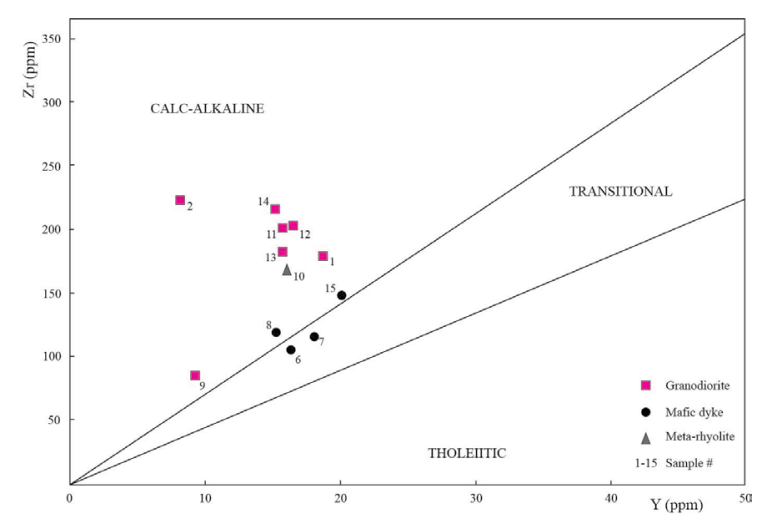

Figure 10. Zr-Y plot classifying samples according to their magma source (after Winchester \& Floyd 1977). Numbers correspond to samples listed in Supplementary Table A1.

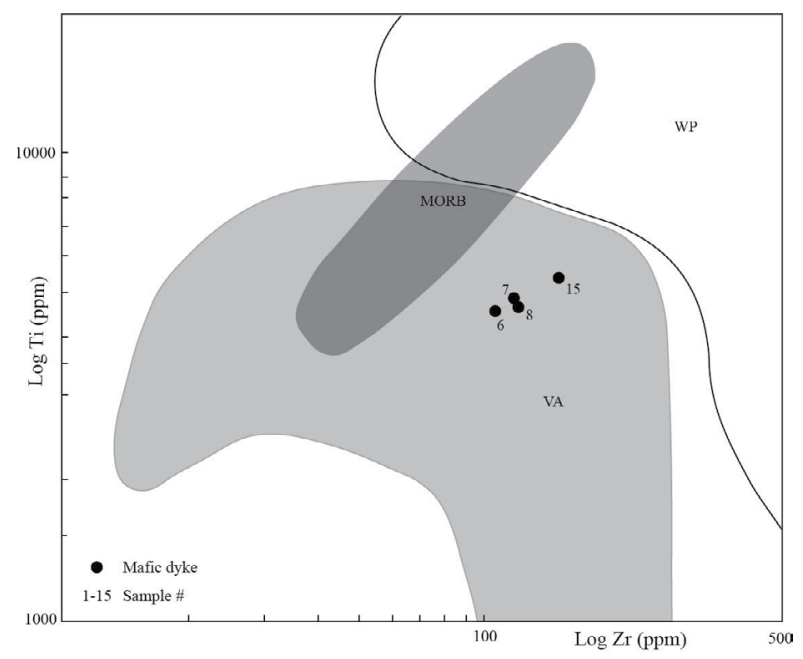

MORB: Mid Ocean Ridge Basalt; VA: volcanic arc; WP: within plate.

Figure 11. $\mathrm{Ti}$ vs. $\mathrm{Zr}$ discrimination diagram (after Pearce 1982). Numbers correspond to samples listed in Supplementary Table A1. density plots and concordia age calculations have been prepared using Isoplot/Ex (Ludwig 2000). The images show that all zircons are magmatically zoned. The rims and cores of ten zircon prismatic crystals were selected for analysis (Fig. 12). A standard concordia plot of ${ }^{206} \mathrm{~Pb} /{ }^{238} \mathrm{U}$ vs. ${ }^{207} \mathrm{~Pb} /{ }^{235} \mathrm{U}$ data (Fig. 13) yields an upper intercept age of $2147 \pm 17 \mathrm{Ma}$, which is interpreted as the maximum age of the intrusion of the granodiorite. Zircon sample YK 1092.1 yielded a younger age of $1988 \pm 22 \mathrm{Ma}$ and is attributed to $\mathrm{Pb}$ loss in the system. The ${ }^{207} \mathrm{~Pb} /{ }^{206} \mathrm{~Pb}$ vs. ${ }^{238} \mathrm{U} /{ }^{206} \mathrm{~Pb}$ plot (Fig. 14) yields the age of $2142 \pm 12 \mathrm{Ma}$, which is interpreted as the minimum age of intrusion of the granodiorite. The mean derived age is estimated at $2146 \pm 15 \mathrm{Ma}$ (Fig. 15). Taken together, these results bracket the timing of intrusion of the 9 Mile Deposit granodiorite between $2142 \pm 15$ and $2147 \pm 17$ Ma.

\section{DISCUSSION AND CONCLUSIONS}

Rare fresh outcrops in the vicinity of the 9 Mile Deposit confirm inferences based on regional syntheses. The host rocks and granodiorite record an evolution typical of greenstone belts in the Guiana Shield that was affected by the Trans-Amazonian orogeny. Although lithogeochemical analyses (Figs. 7 and 11) suggest that the granodiorite and meta-rhyolite are both crustally-derived, field relationships indicate that the meta-rhyolite was metamorphosed prior to the intrusion of the granodiorite, and so they were not co-magmatic. The granodiorite is a local representative of regional syn- to late-tectonic intrusions that characterize the Trans-Amazonian orogeny at ca. $2.15 \mathrm{Ga}$ (Voicu 1999). Field relationships also indicate that the meta-rhyolite is not contemporaneous with the granodiorite and the time that has elapsed between emplacement of the two rocks could be confirmed with U-Pb dating of the meta-rhyolite. The granodiorite intruded between $2142 \pm 17$ and $2147 \pm 12 \mathrm{Ma}$, an age typical of syn- to late collisional felsic magmatism that characterizes the Trans-Amazonian orogeny (Voicu 1999). Other gold-bearing intrusive rocks, most notably the quartz diorite at the Omai Deposit, are dated at 2094 $\pm 1 \mathrm{Ma}$, whereas the meta-volcanic rock it intrudes has been dated at $2120 \pm 2 \mathrm{Ma}$ (Norcross et al. 2000). Assuming the $2002 \pm 5 \mathrm{Ma}$ age of gold mineralization at the nearby Omai deposit is locally representative, the age range of gold mineralization at the 9 Mile Deposit may be bracketed between the $2147 \pm 17 \mathrm{Ma}$ crystallization of the granodiorite, and $2002 \pm 5 \mathrm{Ma}$, i.e., before the maximum age of mineralization of the 
similar gold deposit. Further, the Salamangone gold deposit with a similar host intrusive crystallization age of $2160 \pm 130 \mathrm{Ma}$ and mineralization age of $1830 \pm$ $270 \mathrm{Ma}$ (Nogueira et al. 2000) might also be regionally correlated with the 9 Mile Deposit and part of the same mineralization event of the MKSZ. It would be useful to conduct kinematic analysis on the MKSZ as it may have regional controls on gold mineralization
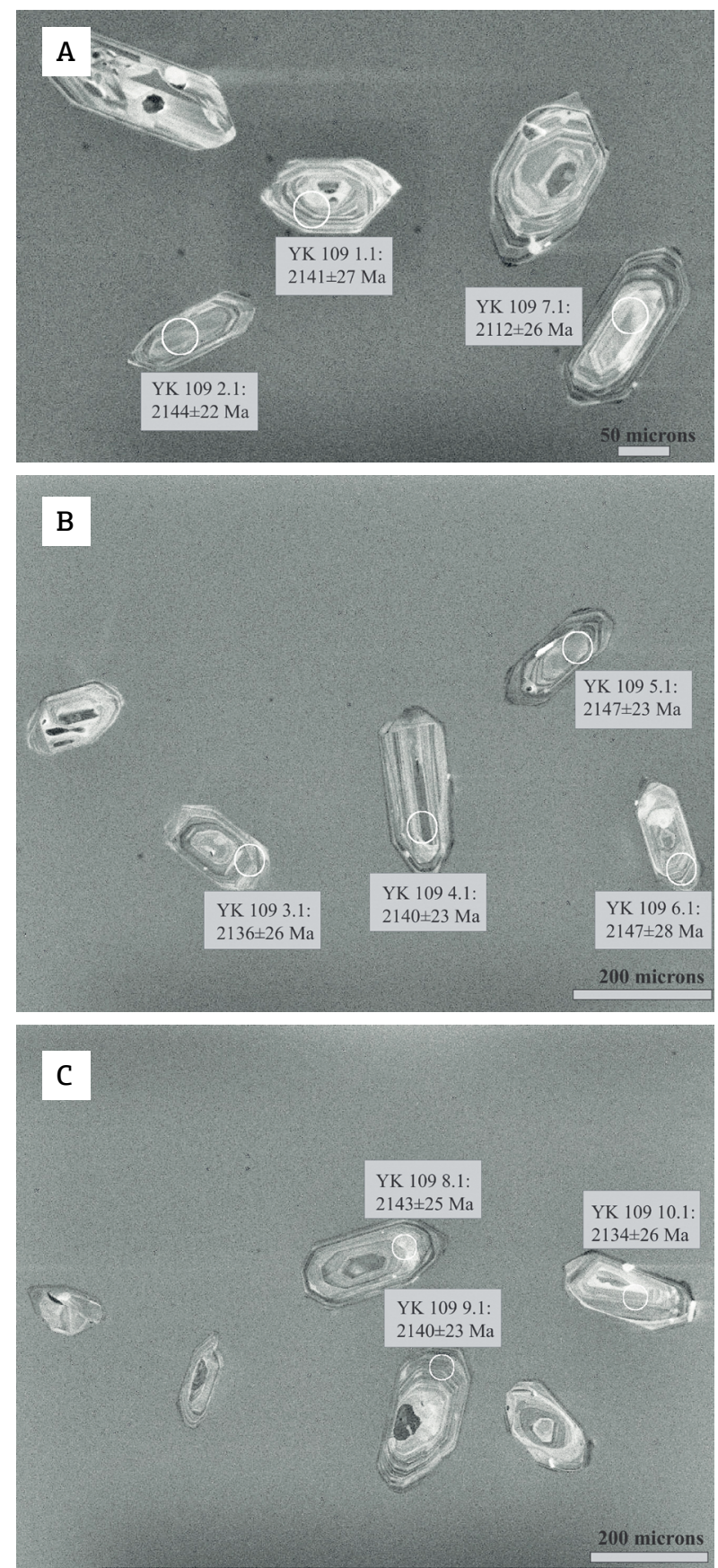

Figure 12. Cathode luminescence zircon images, showing locations of $\mathrm{U}-\mathrm{Pb}$ isotope analyses. The aperture width of the laser is $\mathbf{3 0}$ microns.

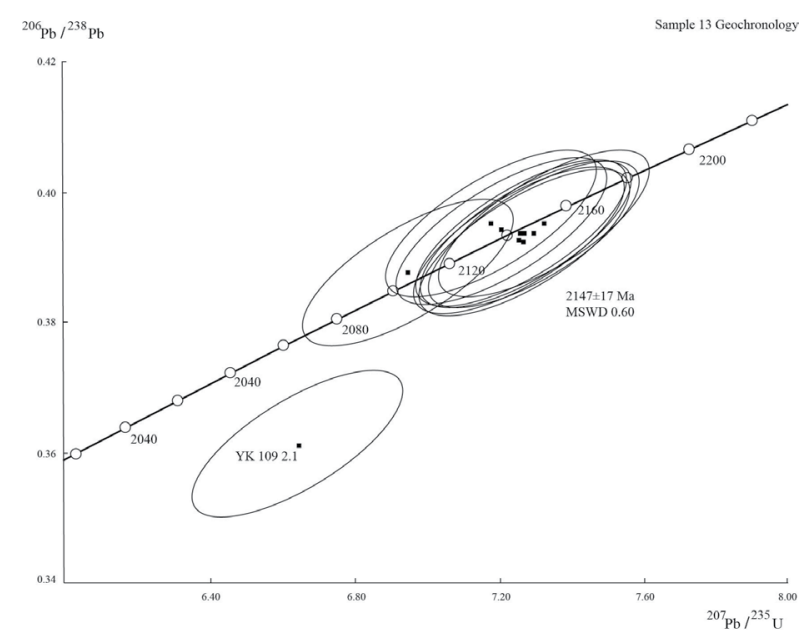

Figure 13. Concordia plot of ${ }^{206} \mathrm{~Pb} /{ }^{238} \mathrm{U}$ vs. ${ }^{207} \mathrm{~Pb} /{ }^{235} \mathrm{U}$ for sample 13.

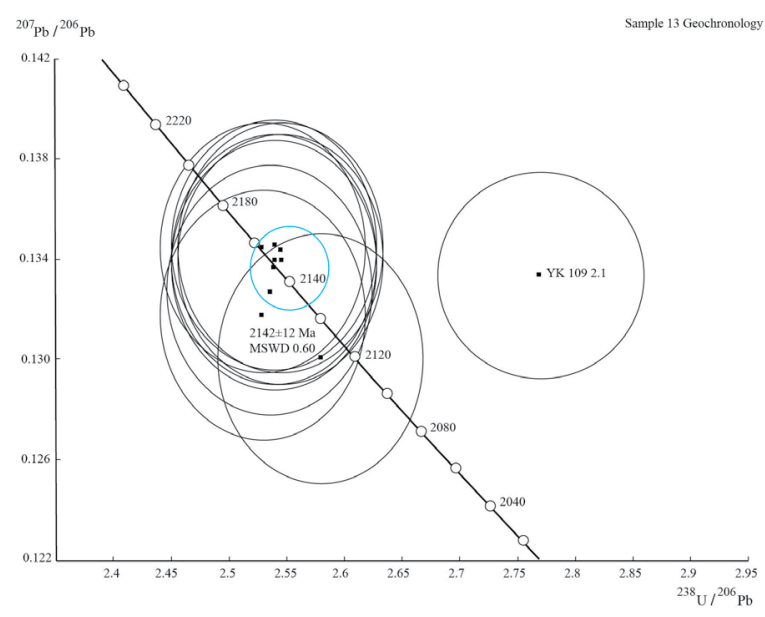

Figure 14. Concordia plot of ${ }^{207} \mathrm{~Pb} /{ }^{206} \mathrm{~Pb}$ vs. ${ }^{238} \mathrm{U} /{ }^{206} \mathrm{~Pb}$ for sample 13 .

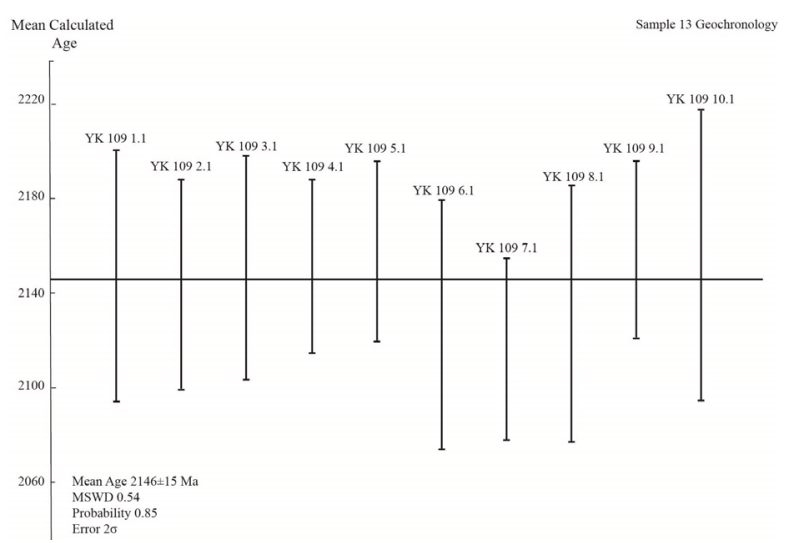

Figure 15. Plot showing weighted mean derived age and error limits of each ${ }^{207} \mathrm{~Pb} /{ }^{206} \mathrm{~Pb}$ ratio for sample 13 . 
in the Guiana Shield. Many gold deposit drill cores are oriented and this could serve as a source of reliable structural measurements for such a study.

\section{ACKNOWLEDGEMENTS}

We thank the reviewers for their constructive comments. Roy Bassoo thanks Hendrik Veldhuyzen for his learned input, Troy Resources and the Perreira Group of Companies for facilitating this study, his wife Michelle and his family. Special thanks is also given to Dr. Kenneth Befus for guidance in preparing the associated figures and tables.

\section{SUPPLEMENTARY DATA}

Supplementary data associated with this article can be found in the online version: Suplementary Figures A1 to $\underline{\text { A10 }}$ and Suplementary Table A1 and A2.

\section{REFERENCES}

Black L.P., Kamo S.L., Allen C.M., Aleinikoff J.N., Davis D.W., Korsch R.J., Foudoulis C. 2003. TEMORA 1: a new zircon standard for U-Pb geochronology. Chemical Geology, 200:155-170. http://doi. org/10.1016/S0009-2541(03)00165-7

Carter J.W., Fernandes L.L. 1966. The stratigraphical, lithological and structural controls to mineralization within the Barama-Mazaruni Assemblage. In: Guiana Geological Conference, 7., Paramaribo, Suriname.

Cordani U.G., Brito Neves B.B. 1982. The geologic evolution of South America during the Archaean and early Proterozoic. Revista Brasileira de Geociências, 12(1):78-88.

Cordani U.G., Teixeira W. 2007. Proterozoic accretionary belts in the Amazonian Craton. Geological Society of America Memoirs, 200:297-320. https://doi.org/10.1130/2007.1200(14)

Daoust C. 2016. Caracterisation stratigraphique, structurale et geochimique du district mineralise de Rosebel (Suriname) dans le Cadre de l'evolution geodynamique de Bouclier Guyanais. PhD Thesis, Universite du Quebec, Montreal.

Daoust C., Voicu G., Brisson H., Gauthier M. 2011. Geological Setting of the Paleoproterozoic Rosebel gold district, Guiana Shield, Suriname. Journal of South American Earth Sciences, 32(3):222-245. http://dx.doi.org/10.1016/j.jsames.2011.07.001

Delor C., Lahondère D., Egal E., Lafon J.M., Cocherie A., Guerrot C., Rossi P., Truffert C., Théveniaut H., Phillips D., de Avelar V.G. 2003. Trans-Amazonian crustal growth and reworking as revealed by the 1:500,000 scale geological map of French Guyana (2nd edition). Geology of France and surrounding areas. Special Guyana Shield. BRGM - SGF, 2-4:5-57.

Elliott R.G. 1992. The geology and the geochemistry of the Omai goldfield, Guyana. Thesis. Oxford Brookes University, Oxford.

Fraga L.M.B., Macambira M.J.B., Dall'Agnol R., Costa J.B.S. 2009. 1.94-1.93 Ga charnockitic magmatism from the central part of the Guyana Shield, Roraima, Brazil: single-zircon evaporation data and tectonic implications. Journal of South American Earth Sciences, 27:247-257. http://doi.org/10.1016/j.jsames.2009.02.007

Frost B.R., Barnes C.G., Collins W.J., Arculus R.J., Ellis D.J, Frost C.D. 2001. A Geochemical Classification for Granitic Rocks. Journal of Petrology, 42(11):2033-2048. https://doi.org/10.1093/ petrology/42.11.2033

Gibbs A.K., Barron C.N. 1993. The Geology of the Guiana Shield. New York: Oxford University Press.

Guiraud G. Tremblay A., Jebrak M. 2017. The Rhyacian "Montagne d'Or" auriferous volcanogenic massive sulphide deposit, French
Guiana, South America: stratigraphy and geochronology. In: SGA Biennial Meeting, 14., Québec, Canada. Proceedings... v. 1.

Kapusta Y. 2014. SHRIMP II analysis report: R. Bassoo, work order A14-3229. Actlabs.

Kroonenberg S.B., De Roever E.W.F., Fraga L.M., Reis N.J., Faraco M.T., Lafon J.M., Cordani U.G., Wong T.E. 2016. Paleoproterozoic evolution of the Guiana Shield in Suriname - a revised model. Netherlands Journal of Geosciences, 95(4):491-522. https://doi.org/10.1017/ njg.2016.10

LaFrance J. 2010. Nine Mile Pit Project: Drilling Exploration Program. Riva Gold Corp report. Riva Gold Corp.

Ludwig K.R. 2000. SQUID 1.00: A User's Manual. Berkeley: Berkeley Geochronology Center Special Publication, n. 2.

McConnell R.B., Williams E. 1970. Distribution and provisional correlation of the Precambrian of the Guiana Shield. In: Guiana Geological Conference, 8., Georgetown, Guiana. Proceedings... p. 1-20.

McDonough W.F., Sun S.S. 1995. The Composition of the Earth. Chemical Geology, 120(3-4):223-253. https://doi. org/10.1016/0009-2541(94)00140-4

Miyashiro A. 1974. Volcanic rock series in island arcs and active continental margin. American Journal of Science, 274(4):321-355. https://doi.org/10.2475/ajs.274.4.321

Nogueira S.A.A., Bettencourt J.S., Tassinari C.C.G. 2000. Geochronology of the granitoid hosted Salamangone Gold Deposit, Lourenco District, Amapa, Brazil. Revista Brasileira de Geociências, 30(2):261-264

Norcross C.E. 1997. U-Pb Geochronology of the Omai Intrusion Hosted Au-Quartz Vein Deposit and Host Rocks, Guiana Shield, South America. Thesis, University of Toronto, Toronto.

Norcross C.E., Davis D.W., Spooner T.C., Rust A. 2000. U-Pb and $\mathrm{Pb}-\mathrm{Pb}$ age constraints on Paleoproterozoic magmatism, deformation and gold mineralization in the Omai area, Guyana Shield. Precambrian Research, 102(1):69-86. http://dx.doi.org/10.1016/ S0301-9268(99)00102-3

Pearce J.A. 1982. Trace element characteristics of lavas from destructive plate boundaries. In: Thorpe R.S. (Ed.), Andesites. New York: Wylie and Sons, p. 525-548.

Pearce J.A., Harris N.B.W., Tindle A.G. 1984. Trace element discrimination diagrams for the tectonic interpretation of granitic rocks. Journal of Petrology, 25:956-983. http://dx.doi.org/10.1093/ petrology/25.4.956\} 
Pearce J.A., Parkinson I.J. 1993. Trace element models for mantle melting: application to volcanic arc petrogenesis. In: Prichard H.M., Alabaster T., Harris N.B.W., Neary C.R. (Eds), Magmatic Processes and Plate Tectonics. Geological Society Special Publications, 76:373-403.

Priem H.N.A., Boelrijk N.A.I.M., Hebeda E.H., Verdurmen E.A., Verschure R.H. 1973. Age of the Precambrian Roraima Formation in Northeastern South America: Evidence from Isotopic Dating of Roraima Pyroclastic Volcanic Rocks in Suriname. GSA Bulletin, 84(5):1677-1684. https:// doi.org/10.1130/0016-7606(1973)84\%3C1677:AOTPRF\%3E2.0.CO;2

Reis N.J., de Faria M.S.G., Fraga L.M., Haddad R.C. 2000. Orosirian calc-alkaline volcanism and the Orocaima event in the northern Amazonian Craton, eastern Roraima State. Revista Brasileira de Geociências, 30(3):380-383.

Santos J.O.S., Potter P.E., Reis N.J., Hartmann L.A., Fletcher I.R., McNaughton N.J. 2003. Age, source and regional stratigraphy of the Roraima Supergroup and Roraima-like outliers in northern South America based on U-Pb geochronology. Geological Society of America Bulletin, 115(3):331-348.

Snelling N.J., McConnell R.B. 1969. The geochronology of Guyana. Geologie en Mijnbouw, 48:201-213.

Tassinari C.C.G. 1997. The Amazonian Craton. In: De Wit M.J., Ashwal M.J. (Eds.), Greenstone Belts. Oxford: Clarendon Press, p. 558-566.

Vanderhaeghe O., Ledru P., Thiéblemont D., Egal E., Cocherie A Tegyey M., Milési J.P. 1998. Contrasting mechanism of crustal growth Geodynamic evolution of the Paleoproterozoic granite greenstone belts of French Guiana. Precambrian Research, 92(2):165-193. http://doi.org/10.1016/S0301-9268(98)00074-6

Veldhuyzen H. 2010. November 2010, Property Visit, 9 Mile Mine, Mazaruni \#3 Mining District, Guyana. Riva Gold Corp Report, 400-837.
Voicu G. 1999. Geology, Geochemistry and Metallogeny of the Omai Gold Deposit, Guiana Shield, South America. PhD thesis, University of Quebec, Montreal.

Voicu G., Bardoux M., Jebrak M., Crepeau R. 1999. Structural Mineralogical and Geochemical Studies of the Paleoproterozoic Omai Gold Deposit, Guyana. Economic Geology, 94(8):1277-1303. https://doi.org/10.2113/gsecongeo.94.8.1277

Voicu G., Bardoux M., Stevenson R. 2001. Lithostratigraphy, geochronology and gold metallogeny in the Northern Guiana Shield, South America: A review. Ore Geology Reviews, 18(3-4):211-236. http://dx.doi.org/10.1016/S0169-1368(01)00030-0

Walrond G.W. 1987. Geological map of Guyana, scale 1:1 million. Georgetown, Guyana: Guyana Geology and Mines Commission.

Wiedenbeck M., Allé P., Corfu F., Griffin W.L., Meier M., Oberli F., von Quadt A., Roddick J.C., Spiegel W. 1995. Three natural zircon standards for U-Th-Pb, Lu-Hf, trace element and REE analyses. Geostandards Newsletter, 19(1):1-23. https://doi.org/10.1111/ j.1751-908X.1995.tb00147.x

Wetherill G.W. 1956. Discordant uranium-lead ages. Transactions American Geophysical Union (American Geophysical Union), 37(3):320-326. https://doi.org/10.1029/TR037i003p00320

Williams I.S. 1998. U-Th-Pb Geochronology by Ion Microprobe. In: McKibben M.A., Shanks III W.C., Ridley W.I. (Eds.), Applications of microanalytical techniques to understanding mineralizing processes. Reviews in Economic Geology, 7:1-35.

Winchester J.A., Floyd P.A. 1977. Geochemical discrimination of different magma series and their differentiation products using immobile elements. Chemical Geology, 20:325-343. https://doi. org/10.1016/0009-2541(77)90057-2 